\title{
Analysis of the Long-Term Prognosis in Japanese Patients with Ulcerative Colitis Treated with New Therapeutic Agents and the Correlation between Prognosis and Disease Susceptibility Loci
}

\author{
Kasumi Hishinuma Rintaro Moroi Daisuke Okamoto Yusuke Shimoyama \\ Masatake Kuroha Hisashi Shiga Yoichi Kakuta Yoshitaka Kinouchi \\ Atsushi Masamune \\ Division of Gastroenterology, Tohoku University Hospital, Sendai, Japan
}

\author{
Keywords \\ Ulcerative colitis · Inflammatory bowel disease $\cdot$ Biologic \\ agents · Biomarkers · Genetic susceptibility
}

\begin{abstract}
Background: New therapeutic agents, including biologics and small-molecule drugs, are widely used to treat ulcerative colitis (UC). This study evaluates long-term prognosis in Japanese patients treated with these agents and the association between prognosis and genetic susceptibility to UC. Methods: We evaluated surgery-free rates using the Kaplan-Meier method in the total cohort and in patients treated with prednisolone and new therapeutic agents. Multivariate analysis was performed to identify clinical factors affecting surgical rates using Cox's proportional hazard model. The rate of use of new therapeutic agents was compared using the KaplanMeier method, and multivariate analysis was conducted to investigate the correlation between the single-nucleotide polymorphism (SNP) rs117506082 and long-term prognosis. Results: Surgery-free survival decreased over time. There was no significant difference in this parameter between pa-
\end{abstract}

karger@karger.com www.karger.com/iid

Karger $\stackrel{\text { ' }}{5}$

GOPEN ACCESS
(C) 2021 The Author(s)

Published by S. Karger AG, Basel

This is an Open Access article licensed under the Creative Commons Attribution-NonCommercial-4.0 International License (CC BY-NC) (http://www.karger.com/Services/OpenAccessLicense), applicable to the online version of the article only. Usage and distribution for commercial purposes requires written permission. tients who were administered prednisolone and those who were administered new therapeutic agents. Poor response to prednisolone and treatment without topical 5-aminosalicylic acid were poor prognostic factors. Shorter time from diagnosis to initiation of treatment with new therapeutic agents was a risk factor for colectomy. The AA genotype of SNP rs 117506082 was associated with a shorter time to surgery and increased use of new therapeutic agents. Conclusions: The use of new therapeutic agents might improve long-term prognosis in patients with more severe UC. Previously identified genetic risk factors were not significantly associated with a higher rate of colectomy.

(c) 2021 The Author(s)
Published by S. Karger AG, Basel

\section{Introduction}

Ulcerative colitis (UC) is a multifactorial inflammatory disorder of the colon that primarily affects the colonic mucosa, forming erosions and ulcers $[1,2]$. It is thought that multiple factors, such as environmental and genetic factors and intestinal flora, are involved in the on- 
Table 1. Clinical characteristics of the study population
Total

$(n=166)$

Gender (male/female)

$107 / 59$

Age at diagnosis, years (median $\pm \mathrm{SD}$ )

$29 \pm 15.4$

Disease location (proctitis/left sided colitis/pancolitis/segmental colitis)

Smoke history, $n$ (\%)

$12 / 39 / 109 / 6$

$53(31.9)$

$16(9.6)$

Family history of IBD, $n(\%)$

$3(1.8)$

$\mathrm{SD}$, standard deviation; IBD, inflammatory bowel disease.

set of inflammatory bowel disease (IBD) [3]. The etiology of UC is unclear, and no curative treatment has been established. Although many UC patients treated with 5-aminosalicylic acid (5-ASA) have a good prognosis, several cases are associated with a severe clinical course requiring systemic steroid therapy, immunosuppressive therapy, and surgical intervention $[1,2,4]$. In recent years, new therapeutic agents, including biologics, tacrolimus, and tofacitinib, have become available worldwide, expanding the therapeutic options for UC [5-12]. In Japan, several therapeutic agents, including tacrolimus, have been approved since 2009 [13]. These agents achieve high remission rates even in steroid-dependent and resistant cases and reduce the need for surgery $[8,14,15]$. However, long-term prognosis in Japanese UC patients treated with these new drugs and the clinical factors that affect prognosis are unclear. Therefore, it is essential to identify long-term outcomes and such clinical factors to establish appropriate treatment strategies.

More than 200 IBD susceptibility loci have been identified in a genome-wide association study (GWAS) [16, 17]. We performed a GWAS in Japanese UC patients using a single nucleotide polymorphism (SNP) array (Japonica array) [18] developed by Tohoku University Medical Megabank Organization in Tohoku, Japan, to determine the genetic background of Japanese IBD patients. In addition, susceptibility polymorphisms strongly associated with UC were identified at the human leukocyte antigen (HLA) region [19]. The top SNP (with the lowest $p$ value) was rs117506082. The G allele of this SNP increases the risk of UC development, whereas the A allele is a reference allele [19]. Furthermore, although the GG genotype of SNP rs117506082 was a risk factor for the onset of UC, the need for surgery in a 30-year follow-up was significantly lower in this genotype than in the GA and AA alleles, suggesting that the GG genotype might have a better clinical outcome. However, the analysis of the re-
Table 2. Details of treatments in the study population (containing duplication)

\begin{tabular}{lc}
\hline & Total $(n=166)$ \\
\hline Systemic steroid, $n(\%)$ & $118(71.1)$ \\
Thiopurine, $n(\%)$ & $85(51.2)$ \\
Leukocytapheresis, $n(\%)$ & $41(24.7)$ \\
New treatments, $n(\%)$ & $81(48.8)$ \\
Tacrolimus, $n(\%)$ & $46(27.7)$ \\
Infliximab, $n(\%)$ & $45(27.1)$ \\
Adalimumab, $n(\%)$ & $25(15.1)$ \\
Golimumab, $n(\%)$ & $12(7.2)$ \\
Tofacitinib, $n(\%)$ & $8(4.8)$ \\
Vedolizumab, $n(\%)$ & $9(5.4)$ \\
Topical 5-ASA, $n(\%)$ & $72(43.4)$ \\
Colectomy, $n(\%)$ & $26(15.7)$ \\
\hline
\end{tabular}

5-ASA, 5-aminosalicylic acid.

lationship between these genotypes and clinical outcomes did not consider the reduced need for surgery because the treatment strategies for UC have changed since the approval of new therapeutic agents. Therefore, it is crucial to assess the effect of the $G$ allele on the clinical outcomes of UC patients treated with these agents. This study evaluates long-term prognosis in Japanese UC patients treated with new therapeutic agents, identifies the factors that affect prognosis, and examines the relationship between prognosis and disease susceptibility loci.

\section{Materials and Methods}

\section{Ethical Considerations}

The study protocol was approved by the Research Ethics Committee of our University (2018-1-138, 2018-1-951) and conformed to the ethical guidelines of the Declaration of Helsinki. Written informed consent was obtained from all patients. 


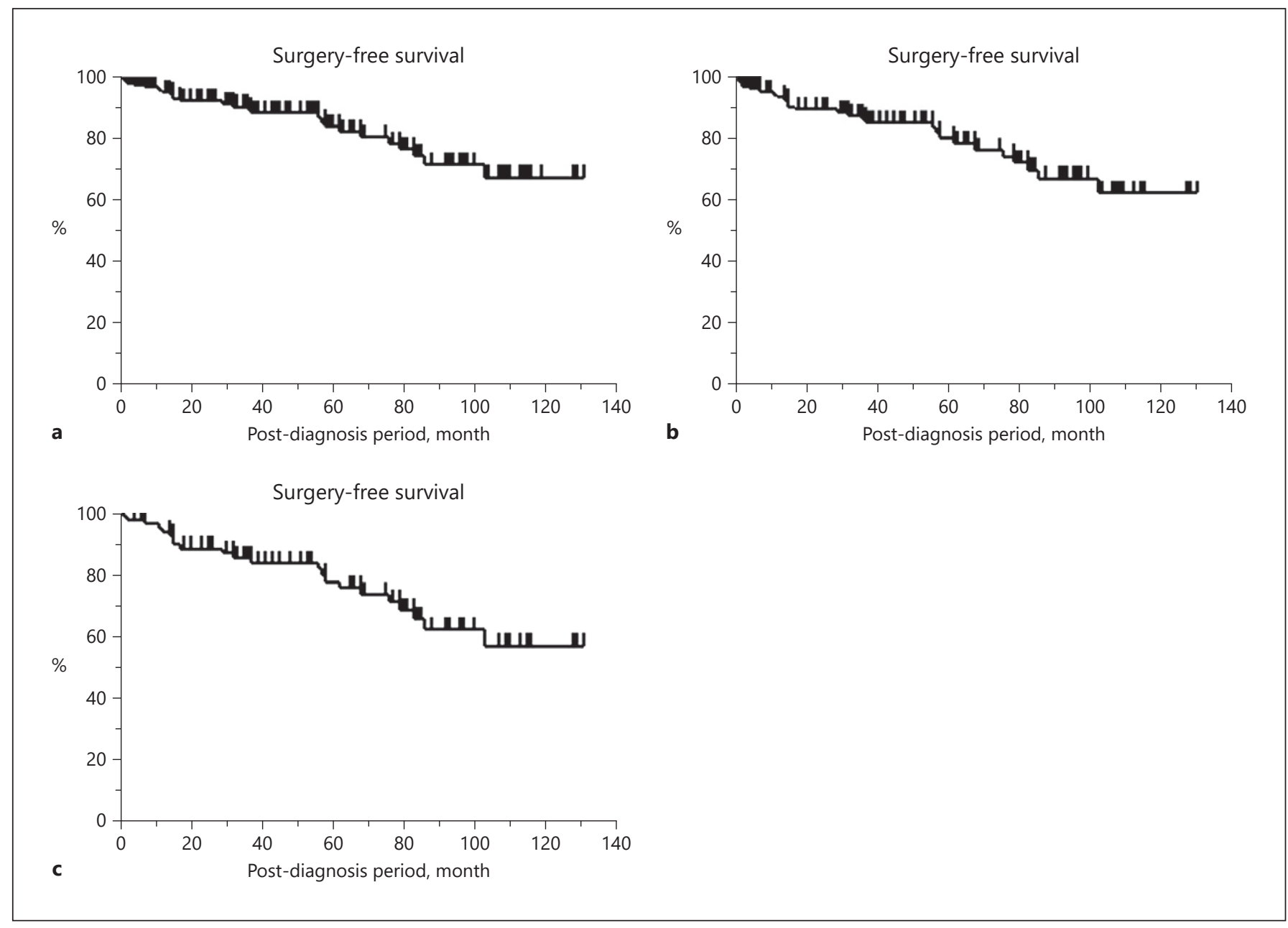

Fig. 1. Surgery-free survival. a Surgery-free survival in the total cohort at 1, 3, and 7 years was $94.8 \%, 89.0 \%$, and $74.0 \%$, respectively. b Surgery-free survival at 1, 3, and 7 years in patients treated with systemic steroids was $92.8 \%$, $85.7 \%$, and $69.4 \%$, respectively. c Surgery-free survival at 1, 3, and 7 years in patients treated with new therapeutic agents was $93.6 \%, 85.3 \%$, and $65.8 \%$, respectively. Censored subjects are indicated by tick marks.

\section{Study Design}

This retrospective observational cohort study was performed at a single center.

\section{Patients}

A total of 166 Japanese patients diagnosed with UC between January 2009 and December 2019 were included in the study.

\section{Evaluation of Long-Term Prognosis}

We reviewed the medical records of patients treated with steroids or new therapeutic agents and evaluated surgery-free survival using the Kaplan-Meier method. The patients who underwent surgery because of colorectal cancer or dysplasia associated with UC were excluded. The cohort included patients not treated with steroids or new therapeutic agents (only 5-ASA or leukocytapheresis therapy).
Identification of Factors Associated with Long-Term Outcomes

The association between clinical factors and long-term prognosis was analyzed using a log-rank test. The investigated clinical factors were sex; age at diagnosis ( $<$ or $\geq 30$ years); disease location (entire colon or other regions); family history of IBD; smoking history; use of topical 5-ASA; response to steroid, thiopurine, and leukocytapheresis therapy; use of new therapeutic agents, including tacrolimus, infliximab, adalimumab, golimumab, tofacitinib, and vedolizumab; and drug-free remission. The cutoff for age at diagnosis was 30 years, which was the median age of the study participants. A multivariate analysis was performed using Cox's proportional hazard model to identify independent risk factors for colectomy.

Analysis of the Association between Genotype and Long-Term Prognosis

The cohort was divided into 2 groups according to the presence or absence of the genotypes GG + GA or AA of SNP rs117506082 at the HLA loci (reference allele: A, alternative allele. Data from 
Table 3. Summary of association between clinical factors and long-term outcome (colectomy) in all study cohorts

\begin{tabular}{|c|c|c|c|c|c|}
\hline \multirow[t]{2}{*}{ Clinical factor } & \multirow[t]{2}{*}{ Patients, $n$} & \multirow{2}{*}{$\frac{\text { Univariate* }^{*}}{p \text { value }}$} & \multicolumn{3}{|c|}{ Multivariate** } \\
\hline & & & HR & $95 \% \mathrm{CI}$ & $p$ value \\
\hline Gender & $\begin{array}{l}\text { Male: } 107 \\
\text { Female: } 59\end{array}$ & 0.29 & $\begin{array}{l}1.24 \\
1\end{array}$ & $0.51-3.02$ & 0.64 \\
\hline Age at diagnosis, years & $\begin{array}{l}<30: 87 \\
\geq 30: 79\end{array}$ & 0.14 & $\begin{array}{l}1 \\
1.73\end{array}$ & $0.76-3.91$ & 0.19 \\
\hline Disease location & $\begin{array}{l}\text { Pancolitis: } 109 \\
\text { Other: } 57\end{array}$ & 0.03 & $\begin{array}{l}1.5 \\
1\end{array}$ & $0.49-4.61$ & 0.48 \\
\hline Response of first systemic steroid & $\begin{array}{l}\text { Responsive: } 90 \\
\text { Resistance: } 24\end{array}$ & 0.029 & $\begin{array}{l}1 \\
3.03\end{array}$ & $1.17-7.86$ & 0.022 \\
\hline New therapeutic agents & $\begin{array}{l}\text { Yes: } 81 \\
\text { No: } 85\end{array}$ & 0.012 & $\begin{array}{l}1.55 \\
1\end{array}$ & $0.51-4.66$ & 0.44 \\
\hline Concomitant topical 5-ASA & $\begin{array}{l}\text { Yes: } 72 \\
\text { No: } 89\end{array}$ & 0.0075 & $\begin{array}{l}1 \\
3.19\end{array}$ & $1.26-8.04$ & 0.014 \\
\hline
\end{tabular}

Alterations in bold indicate statistically significant. CI, confidence interval; 5-ASA, 5-aminosalicylic acid; HR, hazard ratio.

our previous study [19] were used to identify these genotypes in our cohort. Variables including sex, age at diagnosis, disease location, systemic steroid therapy, use of thiopurine and new therapeutic agents, need for colectomy, and time from diagnosis to colectomy were compared between the groups with these 2 genotypes using the $\chi^{2}$ test or Wilcoxon signed-rank test.

The rate of use of new therapeutic agents and surgery-free survival between these 2 groups were determined using the KaplanMeier method. A multivariate analysis was performed using Cox's proportional hazard model to identify independent risk factors for the need for therapeutic agents or colectomy. The investigated clinical factors were age at diagnosis ( $<$ or $\geq 30$ years), disease location (entire colon or other regions), and presence of the genotype GG + GA or AA of SNP rs117506082.

Statistics

The threshold for significance was set at a $p$ value $<0.05$. All analyses were performed using JMP Pro version 14 (SAS Institute Inc., Cary, NC, USA).

\section{Results}

\section{Patient Characteristics}

Clinical characteristics are shown in Table 1. The cohort included 166 patients (109 patients [65.7\%] with pancolitis and 16 patients [9.6\%] with a family history of IBD). The treatment history is summarized in Table 2. A total of $118(71.1 \%)$ and 85 (51.2\%) patients underwent steroid and thiopurine therapy, respectively, and 81 patients $(48.8 \%)$ used the following new therapeutic agents: tacrolimus (46, 27.7\%), infliximab $(45,27.1 \%)$, adalimumab $(25,15.1 \%)$, golimumab $(12,7.2 \%)$, tofacitinib (8, $4.8 \%)$, and vedolizumab $(9,5.4 \%) ; 72$ patients $(43.4 \%)$ used topical 5-ASA.

\section{Overall Long-Term Prognosis}

Surgery-free survival in the total cohort at 1, 3, and 7 years was $94.8 \%, 89.0 \%$, and $74.0 \%$, respectively (Fig. 1a). Surgery-free survival at 1,3 , and 7 years in patients who underwent steroid therapy was $92.8 \%, 85.7 \%$, and $69.4 \%$, respectively (Fig. 1b). Surgery-free survival at 1, 3, and 7 years in patients treated with new therapeutic agents was $93.6 \%, 85.3 \%$, and $65.8 \%$, respectively (Fig. $1 \mathrm{c}$ ).

\section{Factors Associated with Long-Term Outcomes}

The association between clinical factors and the need for surgery is summarized in Table 3. The response to therapy with systemic steroids and topical 5-ASA significantly affected long-term prognosis in the univariate analysis ( $p=0.029$ and 0.0075 , respectively). Multivariate analysis revealed that steroid resistance (hazard ratio [HR]: 3.03, 95\% confidence interval [CI]: 1.17-7.86, $p=$ 0.022 ) and treatment without topical 5-ASA (HR: 3.19, 95\% CI: $1.26-8.04, p=0.014)$ could potentially increase the need for colectomy.

Surgery-free survival was significantly lower in the group with pancolitis (vs. other UC types, $p=0.03$ ) and steroid resistance (vs. steroid sensitivity, $p=0.029$ ), as 


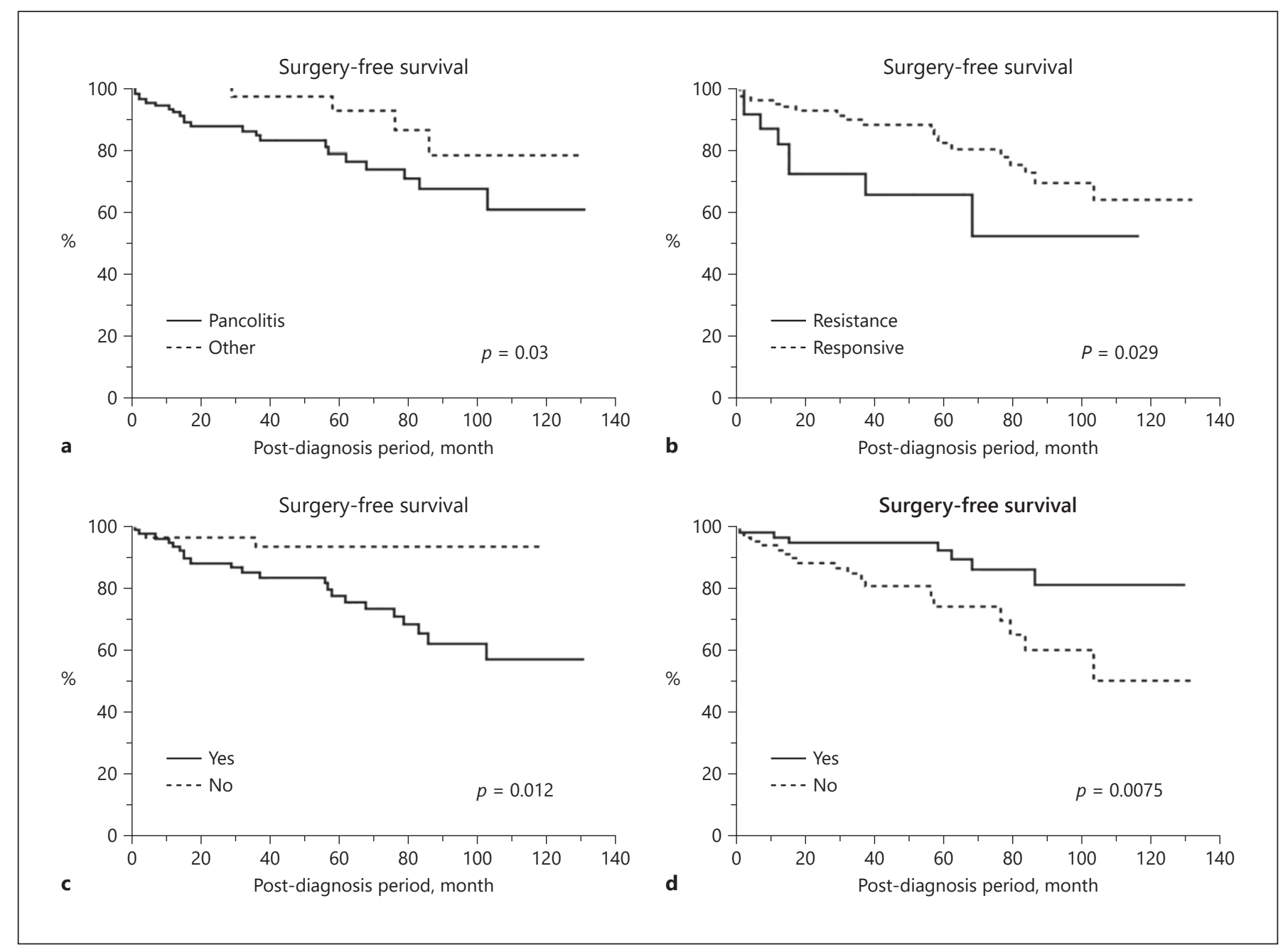

Fig. 2. Surgery-free survival according to clinical factors. a Surgery-free survival was significantly lower in the group with pancolitis (vs. other UC types, $p=0.03$ ). $\mathbf{b}$ Surgery-free survival was significantly lower in the group with steroid resistance (vs. steroid sensitivity, $p=0.029$ ). c Surgery-free survival was significantly lower in the group treated with new therapeutic agents (vs. other agents, $p=0.012$ ). d Surgery-free survival was significantly lower in the group treated without topical 5-ASA (vs. those treated with topical 5-ASA, $p=0.0075$ ). UC, ulcerative colitis; 5-ASA, 5-aminosalicylic acid.

well as in patients treated with therapeutic agents (vs. those not treated with these agents, $p=0.012$ ) and those treated without topical 5-ASA (vs. patients treated with this drug, $p=0.0075$ ) (Fig. 2). We performed a similar analysis in patients with a history of steroid therapy (Table 4). Multivariate analysis showed that steroid resistance (HR: 3.03, 95\% CI: 1.17-7.89, $p=0.022$ ) and treatment without topical 5-ASA (HR: 3.19, 95\% CI: $1.26-$ $8.04, p=0.014$ ) could significantly increase the need for colectomy.

Surgery-free survival was significantly lower in the group with steroid resistance (vs. steroid sensitivity, $p=$
0.029 ) and in the group treated without topical 5-ASA (vs. patients treated with this medication, $p=0.018$ ) (Fig. 3). The association between clinical factors and long-term outcomes in patients treated with therapeutic agents was analyzed (Table 5). Multivariate analysis revealed that the use of therapeutic agents within 16 months after diagnosis was a significant independent risk factor for surgery $(\mathrm{HR}=6.15,95 \% \mathrm{CI}=1.93-19.6, p=0.0021)$. Surgery-free survival was significantly lower in patients treated with therapeutic agents within 16 months after diagnosis (vs. $\geq 16$ months, $p=0.0013$ ) (Fig. 4). 


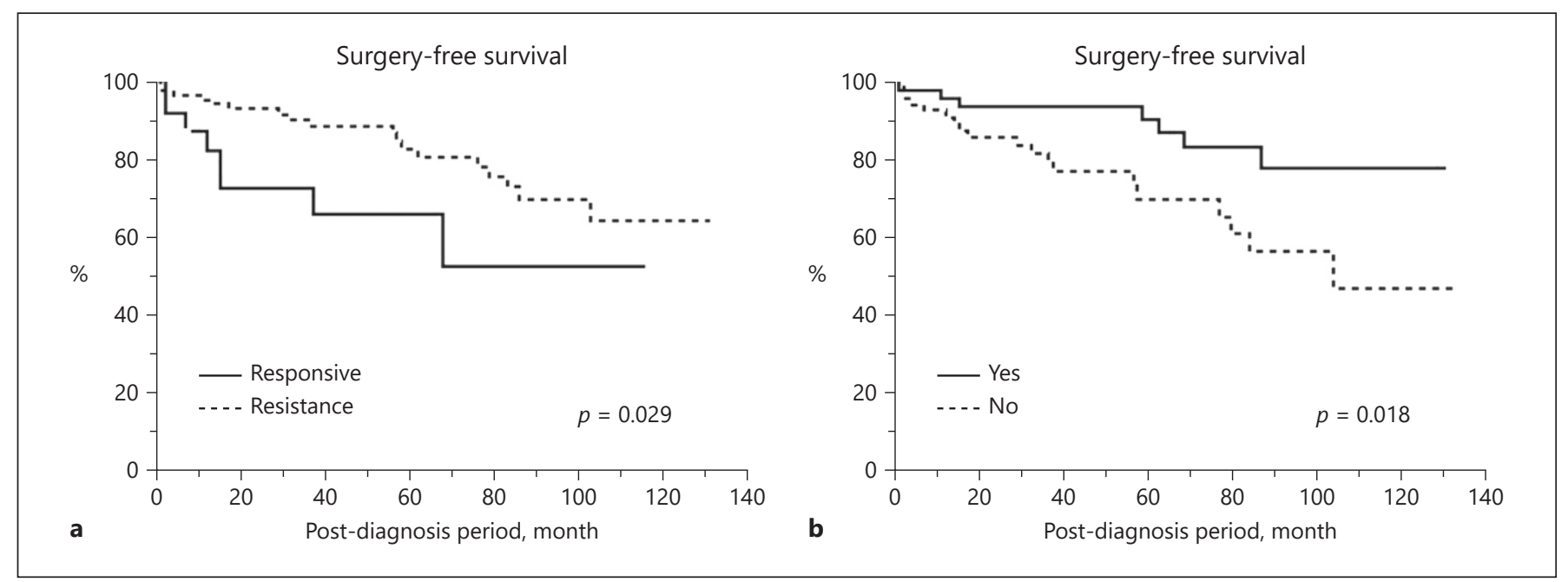

Fig. 3. Surgery-free survival in patients treated with systemic steroids. a Surgery-free survival was significantly lower in the group with steroid resistance (vs. steroid sensitivity, $p=0.029$ ). b Surgery-free survival was significantly lower in the group treated without topical 5-ASA (vs. those treated with topical 5-ASA, $p=0.018$ ). 5-ASA, 5-aminosalicylic acid.

Table 4. Summary of association between clinical factors and long-term outcomes (colectomy) in patients treated with systemic steroids

\begin{tabular}{|c|c|c|c|c|c|}
\hline \multirow[t]{2}{*}{ Clinical factor } & \multirow[t]{2}{*}{ Patients, $n$} & \multirow{2}{*}{$\frac{\text { Univariate* }^{*}}{p \text { value }}$} & \multicolumn{3}{|c|}{ Multivariate** } \\
\hline & & & hazard ratio & $95 \%$ CI & $p$ value \\
\hline Gender & $\begin{array}{l}\text { Male: } 83 \\
\text { Female: } 35\end{array}$ & 0.51 & $\begin{array}{l}1.24 \\
1\end{array}$ & $0.51-3.02$ & 0.64 \\
\hline Age at diagnosis, years & $\begin{array}{l}<30: 56 \\
\geq 30: 62\end{array}$ & 0.25 & $\begin{array}{l}1 \\
1.73\end{array}$ & $0.76-3.91$ & 0.19 \\
\hline Disease location & $\begin{array}{l}\text { Pancolitis: } 89 \\
\text { Other: } 29\end{array}$ & 0.11 & $\begin{array}{l}1.5 \\
1\end{array}$ & $0.49-4.61$ & 0.48 \\
\hline Response of first systemic steroid & $\begin{array}{l}\text { Responsive: } 90 \\
\text { Resistance: } 24\end{array}$ & 0.029 & $\begin{array}{l}1 \\
3.03\end{array}$ & $1.17-7.86$ & 0.022 \\
\hline New therapeutic agents & $\begin{array}{l}\text { Yes: } 80 \\
\text { No: } 38\end{array}$ & 0.23 & $\begin{array}{l}1.55 \\
1\end{array}$ & $0.51-4.66$ & 0.44 \\
\hline Concomitant topical 5-ASA & $\begin{array}{l}\text { Yes: } 47 \\
\text { No: } 70\end{array}$ & 0.018 & $\begin{array}{l}1 \\
3.19\end{array}$ & $1.26-8.04$ & 0.014 \\
\hline
\end{tabular}

5-ASA, 5-aminosalicylic acid; HR, hazard ratio; CI, confidence interval. * Log-rank test; ** Cox's proportional hazard model.

\section{Association between Genotype and Long-Term \\ Prognosis}

We identified the SNP rs117506082 in 87 patients. The characteristics of these patients are shown in Table 6 . There were significant differences in disease location and time from diagnosis to colectomy between the groups with the GG + GA and AA genotype ( $p=0.03$ and 0.042 , respectively). The GG + GA group tended to have less extensive disease and a longer time to surgery.

The rate of use of therapeutic agents was significantly higher, and surgery-free survival was significantly lower in the AA group (vs. the GG + GA group; $p=0.014$ and 
Table 5. Summary of association between clinical factors and long-term outcomes (colectomy) in patients treated with the new therapeutic agents

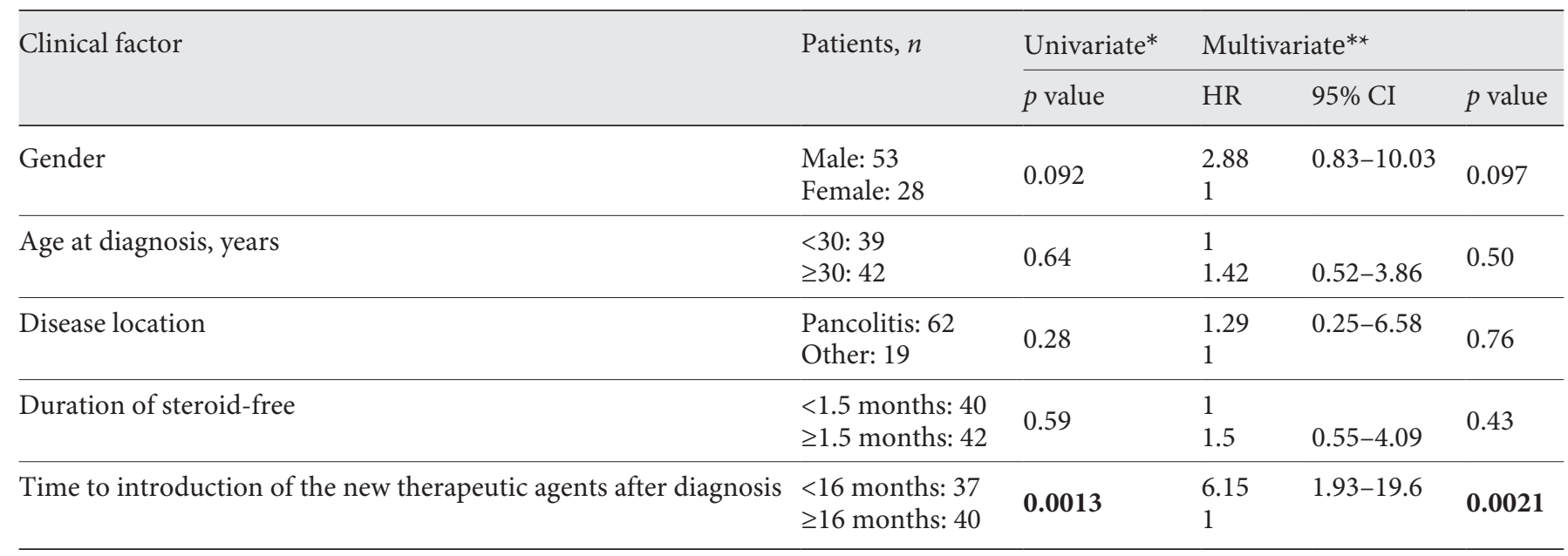

HR, hazard ratio; CI, confidence interval. * Log-rank test; ${ }^{* *}$ Cox's proportional hazard model.

0.028, respectively) (Fig. 5). The results of the multivariate analysis using Cox's proportional hazard model showed that the AA genotype was a significant independent risk factor for the need for therapeutic agents ( $\mathrm{HR}=$ $2.11,95 \% \mathrm{CI}=1.07-4.14, p=0.031$ ) (Table 7).

\section{Discussion}

In this study, we analyzed the role of multiple factors in the long-term prognosis in Japanese UC patients treated with prednisolone and new therapeutic agents. Surgery-free survival decreased over time. The multivariate analysis revealed that a poor response to steroid therapy, treatment without topical 5-ASA, and shorter time from diagnosis to treatment with therapeutic agents increase the need for colectomy. Moreover, the A allele of SNP rs117506082 worsened the prognosis, shortening the time to surgery and increasing the need for therapeutic agents.

Surgery-free survival at 1 and 7 years was $94.5 \%$ and $74 \%$, respectively. This rate decreased over time in all groups, and survival at 7 years was lowest $(69.4 \%)$ in the group with severe UC which required systemic steroid therapy. A few cases required surgery in the long term, even when remission was achieved. This result is consistent with a previous study, wherein the surgical rate decreased in the short term after using tacrolimus or infliximab but raises the concern that the surgical rate may increase in the long term [20]. Thus, predicting prognosis

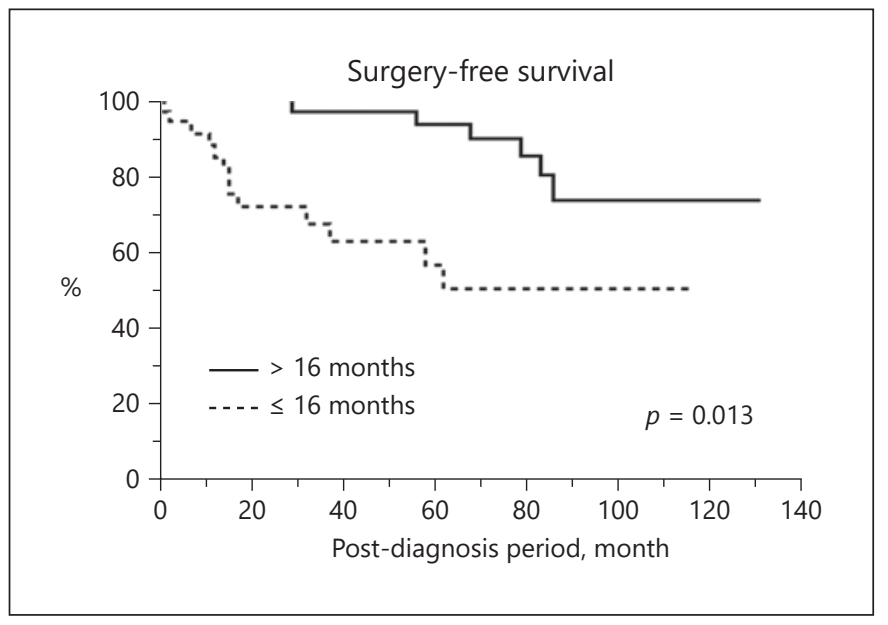

Fig. 4. Surgery-free survival in patients treated with biologic agents. Surgery-free survival was significantly lower in the patients treated with new therapeutic agents within 16 months after diagnosis (vs. $\geq 16$ months, $p=0.0013$ ).

in patients treated with these therapeutic agents is challenging.

In the total cohort, univariate analysis revealed that pancolitis, steroid resistance, the early use of therapeutic agents, and treatment without 5-ASA significantly increased the risk of surgery, and multivariate analysis showed that steroid resistance and treatment without topical 5-ASA significantly increased this risk. A similar tendency was noted in patients treated with systemic steroids. 


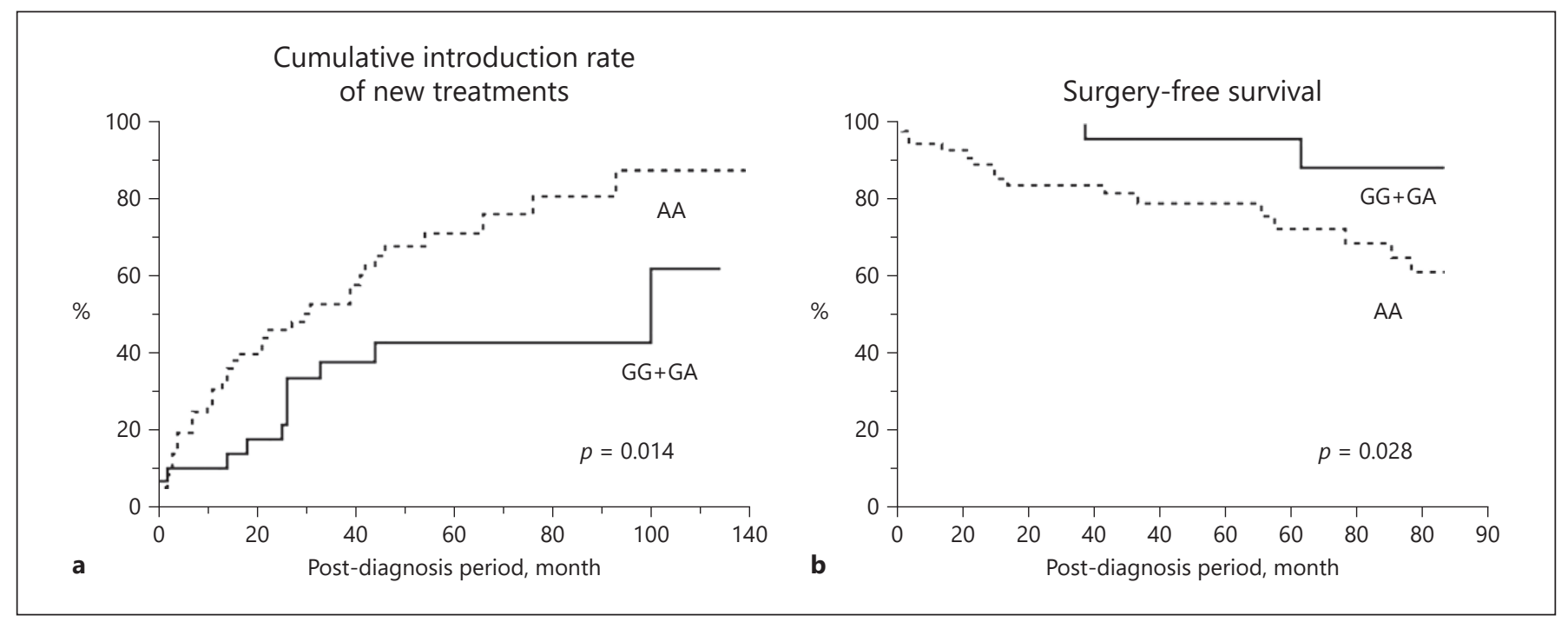

Fig. 5. Rate of use of new therapeutic agents and surgery-free survival according to rs117506082 genotype. a The rate of use of new therapeutic agents was significantly higher in the AA genotype group (vs. the GG + GA genotype group, $p=0.014$ ). $\mathbf{b}$ Surgery-free survival was significantly lower in the AA genotype group (vs. the GG + GA genotype group, $p=0.028$ )

Table 6. Clinical characteristics of the study population based on the rs117506082 genotype

\begin{tabular}{lccc}
\hline & $\begin{array}{l}\text { GG }+\mathrm{GA} \\
(n=29)\end{array}$ & $\begin{array}{l}\text { AA } \\
(n=58)\end{array}$ & $p$ value \\
\hline Gender (male/female) & $17 / 12$ & $34 / 24$ & 1.00 \\
Age at diagnosis, years (median \pm SD) & $32 \pm 14.3$ & $28 \pm 16.1$ & 0.38 \\
Disease location (proctitis/left-sided colitis/pancolitis/segmental colitis) & $1 / 14 / 14 / 0$ & $3 / 11 / 42 / 2$ & $\mathbf{0 . 0 3}$ \\
Systemic steroid, $n(\%)$ & $21(72.4)$ & $48(82.8)$ & 0.28 \\
Thiopurine, $n(\%)$ & $14(48.3)$ & $40(69.0)$ & 0.10 \\
New therapeutic agents, $n(\%)$ & $12(41.4)$ & $38(65.5)$ & $\mathbf{0 . 0 4}$ \\
Colectomy, $n(\%)$ & $4(13.8)$ & $16(27.6)$ & 0.18 \\
Time to colectomy after diagnosis (median \pm SD), month & $74 \pm 27.8$ & $16 \pm 27.1$ & $\mathbf{0 . 0 4 2}$ \\
\hline
\end{tabular}

Table 7. Summary of association between the rs 117506082 genotype and long-term outcomes (multivariate analysis)

\begin{tabular}{|c|c|c|c|c|c|c|c|}
\hline Clinical factor & Patients, $n$ & HR & $95 \% \mathrm{CI}$ & $p$ value* & HR & $95 \% \mathrm{CI}$ & $p$ value* \\
\hline Disease location & $\begin{array}{l}\text { Pancolitis: } 56 \\
\text { Other: } 31\end{array}$ & $\begin{array}{l}1.77 \\
1\end{array}$ & $0.93-3.36$ & 0.081 & $\begin{array}{l}2.22 \\
1\end{array}$ & $0.64-7.74$ & 0.21 \\
\hline rs117506082 genotype & $\begin{array}{l}\text { GG + GA: } 29 \\
\text { AA: } 58\end{array}$ & $\begin{array}{l}1 \\
2.11\end{array}$ & $1.07-4.14$ & 0.031 & $\begin{array}{l}1 \\
3.88\end{array}$ & $0.88-17.1$ & 0.07 \\
\hline
\end{tabular}

HR, hazard ratio; CI, confidence interval. * Cox's proportional hazard model. 
Patients with pancolitis and steroid resistance have poor clinical outcomes. Patients with pancolitis are more likely to be resistant to 5-ASA $[21,22]$ and require intensive therapies, including surgery $[23,24]$. Patients with steroid resistance are also likely to have a poor prognosis. Therefore, step-up therapy with thiopurine or biologics was recommended to these patients by the Toronto consensus [25]. Our study showed that the use of topical 5-ASA might decrease the need for surgery. 5-ASA has anti-inflammatory effects in a dose-dependent manner and is used in high doses for inducing remission, and oral combined with topical therapy might be more effective than oral therapy alone [26]. Therefore, the use of adequate amounts of 5-ASA to treat UC is essential.

In patients treated with therapeutic agents, surgeryfree survival at 1 and 7 years was $93.6 \%$ and $65.8 \%$, respectively. These agents are used to treat moderate to severe disease and patients with steroid dependence [25]. Thus, it can be assumed that the prognosis in this group is worse than that in the untreated group. However, multivariate analysis showed that the use of therapeutic agents was not a significant risk factor for surgery in steroid-treated patients. This result indicates that the use of these agents in intractable cases may improve long-term prognosis in a manner similar to that of responsive patients. A study reported that the rate of surgery for UC decreased in Japan from 2007 to 2017, whereas the rate of use of biologics increased in the same period [27]. However, our findings showed that early treatment with therapeutic agents after diagnosis significantly increased the need for surgery in both univariate and multivariate analyses. Although this result seems to contradict the result of previous studies, these agents are used in patients who are steroid-resistant or -dependent. The early use of new therapeutic agents is positively associated with a more severe disease course, worsening prognosis. Therefore, surgery-free survival was significantly lower in the early-treated group probably because of more severe disease at onset.

The novelty of this study is the analysis of a cohort treated with therapeutic agents that are commercially available since 2009. We examined the correlation between SNP rs117506082 and clinical outcomes in our cohort. Our previous study showed that SNP rs117506082 at the HLA region was significantly associated with UC in the group with the GA + AA genotype [19]. In contrast, the present study revealed that the AA group had a higher proportion of pancolitis. The surgical rate was higher in the group with pancolitis than in the groups with other UC types. Furthermore, the AA group had a significantly higher need for therapeutic agents and a shorter time to surgery. In the multivariate analysis, the AA genotype was associated with an increased need for therapeutic agents and surgery. These results showed that the AA group tended to have more severe disease than the GA + GG group, whereas the $\mathrm{G}$ allele was a risk allele for UC. However, the AA group had more extensive disease than the GA + GG group and lower surgery-free survival, indicating that disease severity and extent might determine prognosis. We found a relationship between HLA polymorphisms and the clinical course of UC, suggesting that the genotype may be a prognostic marker in UC. Larger prospective studies on longterm prognosis, including disease status, therapeutic response, and surgery rate, are necessary to examine the role of the genotype as a prognostic marker for UC.

After the introduction of biologic therapy, the rate of colectomy decreased [27], whereas postoperative morbidity and the number of patients who required at least 3 surgical procedures increased [28]. Furthermore, the deterioration of the general condition caused by prolonged medical treatment before colectomy increases the risk of sepsis and decreases survival [29]. Therefore, timely referral for surgery or the initiation of biologic therapy should be considered. For instance, in patients with risk factors, including pancolitis, steroid resistance, early indication for biologic therapy, and patients with the AA genotype, elective surgery can be performed when the risk of postoperative complications is decreased by reducing inflammation using biologic agents. Elective surgery during the quiescent phase may decrease the risk of relapse and perioperative complications, improve the postoperative quality of life, and reduce treatment costs. The therapeutic approach should be based on the clinical and the social status of the patient.

This study has limitations. First, the sample size was small and the study group was heterogeneous because of the single-center retrospective nature of the study. Second, our study included a higher number of severe cases than other studies because our center is a tertiary care emergency department. Therefore, our data may not accurately reflect the general UC population. Third, data on endoscopic, pathologic, and blood examinations for evaluating disease severity and treatment effectiveness were not obtained because of the type of experimental design.

\section{Conclusion}

Even after using new therapeutic agents, surgery-free survival decreased over time, and approximately $30 \%$ of our cohort needed surgery. Therefore, it is necessary to 
assess disease location and treatment response at UC onset and develop a treatment strategy to improve surgical planning. HLA gene polymorphism is associated with disease onset and affects surgical rates and the need for therapeutic agents, suggesting that this polymorphism can be used as a prognostic marker in UC.

\section{Statement of Ethics}

The study protocol was approved by the Research Ethics Committee of our institution (2018-1-138, 2018-1-951) and conformed to the ethical guidelines of the Declaration of Helsinki. Written informed consent was obtained from all patients.

\section{Funding Sources}

This work was supported by JSPS KAKENHI grant number JP15H04805 to Y. Kakuta.

\section{Author Contributions}

Kasumi Hishinuma and Rintaro Moroi acquired and analyzed data, performed statistical analysis, and wrote the manuscript; Daisuke Okamoto obtained and interpreted data and performed statistical analysis; Yusuke Shimoyama, Masatake Kuroha, Hisashi Shiga, and Yoichi Kakuta acquired data; Yoshitaka Kinouchi obtained data and revised the manuscript; and Atsushi Masamune revised the manuscript. All authors read and approved the final manuscript.

\section{Data Availability Statement}

The authors declare no conflicts of interest.
The datasets generated during and/or analyzed during the current study are available from the corresponding author on reasonable request.

\section{References}

1 Ko CW, Singh S, Feuerstein JD, Falck-Ytter C, Falck-Ytter Y, Cross RK, et al. AGA clinical practice guidelines on the management of mild-to-moderate ulcerative colitis. Gastroenterology. 2019 Feb;156(3):748-64.

2 Feuerstein JD, Isaacs KL, Schneider Y, Siddique SM, Falck-Ytter Y, Singh S, et al. AGA clinical practice guidelines on the management of moderate to severe ulcerative colitis. Gastroenterology. 2020 Apr;158(5):1450-61.

3 Baumgart DC, Carding SR. Inflammatory bowel disease: cause and immunobiology. Lancet. 2007 May 12;369(9573):1627-40.

4 Truelove SC, Witts LJ. Cortisone in ulcerative colitis; final report on a therapeutic trial. $\mathrm{Br}$ Med J. 1955 Oct 29;2(4947):1041-8.

5 Fellermann K, Tanko Z, Herrlinger KR, Witthoeft T, Homann N, Bruening A, et al. Response of refractory colitis to intravenous or oral tacrolimus (FK506). Inflamm Bowel Dis. 2002 Sep;8(5):317-24.

6 Rutgeerts P, Sandborn WJ, Feagan BG, Reinisch W, Olson A, Johanns J, et al. Infliximab for induction and maintenance therapy for ulcerative colitis. N Engl J Med. 2005 Dec 8; 353(23):2462-76

7 Ogata H, Matsui T, Nakamura M, Iida M, Takazoe M, Suzuki Y, et al. A randomised dose finding study of oral tacrolimus (FK506) therapy in refractory ulcerative colitis. Gut. 2006 Sep;55(9):1255-62.

8 Sandborn WJ, Rutgeerts P, Feagan BG, Reinisch W, Olson A, Johanns J, et al. Colectomy rate comparison after treatment of ulcerative colitis with placebo or infliximab. Gastroenterology. 2009 Oct;137(4):1250-520.
9 Sandborn WJ, van Assche G, Reinisch W, Colombel JF, D'Haens G, Wolf DC, et al. Adalimumab induces and maintains clinical remission in patients with moderate-to-severe ulcerative colitis. Gastroenterology. 2012 Feb; 142(2):257-65 e13.

10 Feagan BG, Rutgeerts P, Sands BE, Hanauer S, Colombel JF, Sandborn WJ, et al. Vedolizumab as induction and maintenance therapy for ulcerative colitis. N Engl J Med. 2013 Aug 22;369(8):699-710.

11 Sandborn WJ, Feagan BG, Marano C, Zhang $\mathrm{H}$, Strauss R, Johanns J, et al. Subcutaneous golimumab maintains clinical response in patients with moderate-to-severe ulcerative colitis. Gastroenterology. 2014 Jan;146(1): 96-e1.

12 Sandborn WJ, Su C, Sands BE, D'Haens GR, Vermeire S, Schreiber S, et al. Tofacitinib as induction and maintenance therapy for ulcerative colitis. N Engl J Med. 2017 May 4; 376(18):1723-36.

13 Matsuoka K, Kobayashi T, Ueno F, Matsui T, Hirai F, Inoue N, et al. Evidence-based clinical practice guidelines for inflammatory bowel disease. J Gastroenterol. 2018 Mar;53(3):30553.

14 Rizzo G, Pugliese D, Armuzzi A, Coco C. Anti-TNF alpha in the treatment of ulcerative colitis: a valid approach for organ-sparing or an expensive option to delay surgery? World J Gastroenterol. 2014 May 7;20(17):4839-45.
15 Rungoe C, Langholz E, Andersson M, Basit S, Nielsen NM, Wohlfahrt J, et al. Changes in medical treatment and surgery rates in inflammatory bowel disease: a nationwide cohort study 1979-2011. Gut. 2014 Oct;63(10): 1607-16.

16 Jostins L, Ripke S, Weersma RK, Duerr RH, McGovern DP, Hui KY, et al. Host-microbe interactions have shaped the genetic architecture of inflammatory bowel disease. Nature. 2012 Nov 1;491(7422):119-24.

17 Liu JZ, van Sommeren S, Huang H, Ng SC, Alberts R, Takahashi A, et al. Association analyses identify 38 susceptibility loci for inflammatory bowel disease and highlight shared genetic risk across populations. Nat Genet. 2015 Sep;47(9):979-86.

18 Kawai Y, Mimori T, Kojima K, Nariai N, Danjoh I, Saito R, et al. Japonica array: improved genotype imputation by designing a population-specific SNP array with 1,070 Japanese individuals. J Hum Genet. 2015 Oct;60(10): 581-7.

19 Okamoto D, Kawai Y, Kakuta Y, Naito T, Torisu T, Hirano A, et al. Genetic analysis of ulcerative colitis in Japanese individuals using population-specific SNP array. Inflamm Bowel Dis. 2020 Jul 17;26(8):1177-87.

20 Rodriguez-Lago I, Castro-Poceiro J, Fernandez-Clotet A, Mesonero F, Lopez-Sanroman A, Lopez-Garcia A, et al. Tacrolimus induces short-term but not long-term clinical response in inflammatory bowel disease. Aliment Pharmacol Ther. 2020 May;51(9):8709. 
21 Sicilia B, Vicente R, Arroyo MT, Arribas F, Gomollon F. Ulcerative pancolitis predicts the need for colectomy: study of an incident cohort of patients with ulcerative colitis in Aragon (Spain). Gastroenterol Hepatol. 2005 Feb;28(2):55-9.

22 Lee HJ, Jung ES, Lee JH, Hong SP, Kim TI, $\mathrm{Kim} \mathrm{WH}$, et al. Long-term clinical outcomes and factors predictive of relapse after 5-aminosalicylate or sulfasalazine therapy in patients with mild-to-moderate ulcerative colitis. Hepatogastroenterology. 2012 Jul-Aug; 59(117): 1415-20.

23 Alkim C, Alkim H, Dağli U, Parlak E, Ulker A, Sahın B. Extension of ulcerative colitis. Turk J Gastroenterol. 2011 Aug;22(4):382-7.
24 Kiss LS, Lakatos PL. Natural history of ulcerative colitis: current knowledge. Curr Drug Targets. 2011 Sep;12(10):1390-5.

25 Bressler B, Marshall JK, Bernstein CN, Bitton A, Jones J, Leontiadis GI, et al. Clinical practice guidelines for the medical management of nonhospitalized ulcerative colitis: the Toronto consensus. Gastroenterology. 2015 May; 148(5):1035-e3.

26 Singh S, Feuerstein JD, Binion DG, Tremaine WJ. AGA technical review on the management of mild-to-moderate ulcerative colitis. Gastroenterology. 2019 Feb;156(3):769-e29.
27 Uchino M, Ikeuchi H, Hata K, Okada S, Ishihara S, Morimoto K, et al. Changes in the rate of and trends in colectomy for ulcerative colitis during the era of biologics and calcineurin inhibitors based on a Japanese nationwide cohort study. Surg Today. 2019 Dec;49(12): 1066-73.

28 Abelson JS, Michelassi F, Mao J, Sedrakyan A, Yeo $\mathrm{H}$. Higher surgical morbidity for ulcerative colitis patients in the era of biologics. Ann Surg. 2018 Aug;268(2):311-7.

29 Andrew RE, Messaris E. Update on medical and surgical options for patients with acute severe ulcerative colitis: what is new? World J Gastrointest Surg. 2016 Sep 27;8(9):598-605. 